\title{
Black Hole Superradiance in the Presence of Lorentz Symmetry Violation
}

\author{
Mohsen Khodadi* \\ School of Astronomy, \\ Institute for Research in Fundamental Sciences (IPM) \\ P. O. Box 19395-5531, Tehran, Iran
}

(Dated: March 8, 2021)

\begin{abstract}
In this paper we consider the massive scalar perturbation on the top of a small spinninglike black hole in context of Einstein-bumblebee modified gravity in order to probe the role of spontaneous Lorentz symmetry breaking on the superradiance scattering and corresponding instability. We show that at the low-frequency limit of the scalar wave the superradiance scattering will be enhanced with the Lorentz-violating parameter $\alpha<0$ and will be weakened with $\alpha>0$. Moreover, by addressing the black hole bomb issue, we extract an improved bound in the instability regime indicating that $\alpha<0$ increases the parameter space of the scalar field instability, while $\alpha>0$ decreases it.
\end{abstract}

PACS numbers: 04.70.-s, 04.70.Bw, 04.50.Kd

\section{INTRODUCTION}

Thanks to recent observations stellar-mass black hole collisions by ground-based gravitational-wave detectors [1-3] and capturing the first images of the supermassive black hole's shadow at the center of galaxy M87* by the Event Horizon Telescope (EHT) [4, 5], lots of attentions from astronomers around the world have been attracted to reconsider the physics of black hole more deeply. Newly, also has discovered a stellar-mass black hole which is closer to our Solar System than any other found to date so that by forming part of a triple system it surprisingly can be seen with the naked eye [6]. It is known among astrophysicists that the supermassive black holes are the most likely driving power of spectacular jets in high energetic sources such as active galactic nuclei (AGN) ${ }^{1}$ and quasars at the center of the many galaxies $[8,9]$. However, it should be noted that black holes in nature are not an isolated system rather prone to destroy due to some external perturbations. Therefore, it is wellmotivated to stability analysis of black holes exposed to matter fields in high energy astrophysics. In this regard, one of the mysterious phenomena widely used in the domain of modern physics, is named "superradiance". Generally speaking, we expect this phenomenon reveals in astrophysical black holes belong to the Kerr-Newman family with three characteristic parameters mass, charge and spin. Black hole superradiance actually is an amplification of scattered test field off the rotating and/or charged black hole's horizon which due to energy extracting from background then the reflection coefficient of the wave equation solution to exceed the unit, see leading papers [10]-[18 $]^{2}$. This phenomenon in a sense categorized into black hole perturbation issues so that it occurs just for the bosonic fields perturbations (scalar, electromagnetic and gravitational waves) and not for the massive or massless fermionic test

*Electronic address: m.khodadi@ipm.ir

${ }^{1}$ A new study [7] claims that AGNs actually maybe wormhole mouths rather than supermassive black holes.

${ }^{2}$ If we want to be precise, we have to say that the first treatment of superradiance though wrongly, comes from the seminal work of Klein [19] in the context of non-relativistic quantum mechanics. 
fields such as neutrinos, [20-22]. An impressive message that black hole superradiance has to us is that in the curved spacetime, one can expect to energy extraction from the vacuum via a quite classic process, without regard to any quantum considerations. This is due to this fact that the existence of an event horizon leads to the emergence of an essential ingredient for this phenomenon, namely an intrinsic dissipative mechanism in vacuum [23].

Concerning the Kerr black hole, the energy extraction results in decreasing whether two characteristic parameters mass and spin without violating the second law of black hole known as area law ${ }^{3}$ [24]. As a consequence, using direct relation between black hole area and entropy [25], it was found that in superradiance phenomenon, we just deal with rotational energy extraction from the black hole, not information. So, this phenomena has not any conflict with the event horizon's concept as a one-way membrane which means that there is not information transfer from the interior region to the exterior region of a black hole. There is also another classical mechanism for extracting energy from Kerr black hole known as "Penrose process". It actually was realized earlier than black hole superradiance through Penrose's Gedankenexperiment in which a mass of the matter decomposed into ergoregion so that one of which obtains negative energy, while the other escapes to infinity with more energy than before decomposition ${ }^{4}[26]$. However, it was understood that this process phenomenologically can not be a favor energy extraction mechanism in the sens that it requires an accurate timed breakup of the incoming relativistic particles [13].

Because of transferring energy from black hole to bosonic test field, superradiance phenomenon can generate some instabilities in the background due to traping the superradiant modes near the black hole. More precisely, these instabilities leads to a new phenomenon calling "black hole bomb" because of growing the trapping superradiant modes exponentially between black hole and a turning point. There are some studies in both the contexts of charged and rotating black holes indicating that the turning point can be created generally in two ways. First, it can sourceed by massive scalar field or asymptotically Anti-de-site spacetime, second due to placing a reflective surface like a mirror or cavity around the black hole, see [28]-[47] and references therein. Concerning the second case, may raise the inquiry in mind that is it applicable in realistic situations? The ionized matters such as plasma or accretion disc around astrophysical black holes due to their ability in reflecting of low-frequency electromagnetic waves, are prone to playing the role of the mirror [23]. In some cases the superradiant instabilities may lead to emerging exotic black hole solutions with additional parameters violating the no-hair hypothesis ${ }^{5}$, [51]-[55].

Although during the last century GR have passed many tests with great success and it has been admitted as the standard theory of gravity, still there are some doors to make alternative theories to GR to consider physical phenomena at extremely and small scales ${ }^{6}$. As an important factor from a theoretical viewpoint, having an ultraviolet completion of GR is severely favorable. From observational point of view, GR is not able to describe some gravitational phenomena at the large scale such as the

${ }^{3}$ Note that black hole's area law is valid just for black holes perturbed by matter fields satisfying the weak energy condition. For this reason, one usually does not expect this law works for the black holes subject to the fermionic field perturbations.

4 To have a detailed study of these two energy extraction processes, we refer the interested reader to [27] and also review paper [23]

${ }^{5}$ In recent years, there have been extensive attempts to test the validity of the no-hair hypothesis via confronting its variety statements with some observations, see [48-50] for instance.

${ }^{6}$ In [56] one can find more discussions about different motivations of going beyond Einstein gravity. 
dark side of the universe and seems that something fundamental is still missing.

Black holes can be used a useful probe in the strong regime of gravity in order to check possible high-energy modifications to GR. The imprint of these modifications can leave some signatures in astrophysical phenomena such as strong gravity effects happening in the vicinity celestial bodies like astrophysical black holes and neutron stars. So it is of fundamental importance to investigate astrophysical attributes of black hole in alternative theories of gravity. Given that the astrophysical phenomenon under our attention i.e. black hole superradiance highly is sensitive to underlying geometries, hence in recent years, we have witnessed many types of research about it and its side issues within the extended framework of modified gravity theories, see [57]-[71].

In continuing this, we are interested in studying quench or favor superradiance of the spinning black holes in an extended Lorentz-violating gravity known as the "Einstein-bumblebee model" (EBM) [72]. EBM, contains the innovative idea "spontaneous Lorentz symmetry breaking" (LSB) which actually comes from one of the popular theoretical frameworks to solve quantum gravity issue i.e. string theory. It is worth mentioning to note that the Lorentz symmetry as the fundamental underlying symmetry of two successful field theories describing the universe i.e. GR and the particle standard model, may break at quantum gravity scales. The LSB's idea, has resulted in emergence of an effective field theory known as "standard model extension" (SME) which via it the particle standard model plus GR and every operator may break the Lorentz symmetry, will bring together in one framework [73]-[76]. SME let one for further look for the LSB in multiple frameworks such as: high energy particle physics and astrophysics. SME can be used in most modern analyses of experimental results. EBM is actually one of simple models belong to LSB in which the Lorentz symmetry dynamically breaks by an axial vector field known as "bumblebee field". In other words, the presence of LSB in a local Lorentz frame usually is diagnosed through a nonzero vacuum value for one or more quantities carrying local Lorentz indices which specifically in EBM it is signaled via the bumblebee field. In recent years, we see a remarkable enthusiasm among people for study of interesting physical themes in the framework of EBM, see as example [77]-[90], along with references therein. However, in the meantime, can be seen a vacancy of studying the black hole superradiance. This research is potentially valuable in the sense that due to going to the quantum gravity realm via black holes then it is possible to take the contribution of LSB into superradiance phenomenon.

Aiming to probe the role of LSB on the black hole superradiance phenomenon (in particular amplification factor and superradiance frequency parameters region) as well as the instability related to it, we have organized this paper as follows. In section II, we provide a short review of the derivation of the exact Kerr-like black hole solution in the EBM, based on [85]. In section III, by restoring to a semi-analytically method we release the superradiance amplification factor of bumblebee Kerr-like black hole subject to a massive scalar perturbation. Subsequently we move to investigation on the superradiant instability of underlying system in section IV. Finally, we close our paper with a summarize of concluding discussions and results in section V. Across this work, we use the natural units $c=G=\hbar=1$.

\section{EXACT KERR-LIKE BLACK HOLE SOLUTION IN EINSTEIN-BUMBLEBEE MODEL}

Here, without going to into details we will have a reviwe of the EBM-based Kerr-like black hole solution which recently released in [85]. Bumblebee theory of gravity as a subclass of aether models in 
which Lorentz symmetry is spontaneously broken due to a nonzero vacuum expectation value of the bumblebee vector field $B_{\mu}$ and a proper choosing of potential as well, generally describe by the action $[72]$

$$
S_{E B}=\int d^{4} x \sqrt{-g}\left(\frac{1}{2 \kappa}\left(R+\chi B^{\mu} B^{\nu} R_{\mu \nu}\right)-\frac{1}{4} B^{\mu \nu} B_{\mu \nu}-V\left(B^{\mu} B_{\mu} \pm b^{2}\right)\right), \quad \kappa \equiv 8 \pi
$$

where $\chi$ and $b^{2}$, are respectively the real coupling and numerical constants so that the former is indeed responsible for controlling the non-minimal coupling between the Ricci curvature $R_{\mu \nu}$ and bumblebee field $B_{\mu}$. In the above action, $B_{\mu \nu}$ is named bumblebee field strength and enjoys $U(1)$ gauge invariant, i.e. $B_{\mu \nu}=\partial_{\mu} B_{\nu}-\partial_{\nu} B_{\mu}$. Actually, in this setup the Lorentz symmetry is broken via breaking of the $U(1)$ symmetry of the bumblebee field potential i.e. the collapse of $V$ into a non-zero minimum at $B^{\mu} B_{\mu} \pm b^{2}=0$ and $V^{\prime}\left(b_{\mu} b^{\mu}\right)=0$, with $b^{\mu}=\left\langle B^{\mu}\right\rangle$ which has constant magnitude $b_{\mu} b^{\mu}=\mp b^{2}$. Note that here the prime symbol refers to the differentiation with respect to $b_{\mu} b^{\mu}$.

Concerning the action (2.1), the gravitational field equation in vacuum takes the following form

$$
R_{\mu \nu}=\kappa T_{\mu \nu}^{B}+2 \kappa g_{\mu \nu} V+\frac{1}{2} \kappa g_{\mu \nu} B^{\alpha \beta} B_{\alpha \beta}-\kappa g_{\mu \nu} B^{\alpha} B_{\alpha} V^{\prime}+\frac{\chi}{4} g_{\mu \nu} \nabla^{2}\left(B^{\alpha} B_{\alpha}\right)+\frac{\chi}{2} g_{\mu \nu} \nabla_{\alpha} \nabla_{\beta}\left(B^{\alpha} B^{\beta}\right),
$$

with the bumblebee energy momentum tensor $T_{\mu \nu}^{B}$ as

$$
\begin{aligned}
& T_{\mu \nu}^{B}=-B_{\mu \alpha} B_{\nu}^{\alpha}-\frac{1}{4} g_{\mu \nu} B^{\alpha \beta} B_{\alpha \beta}-g_{\mu \nu} V+2 B_{\mu} B_{\nu} V^{\prime} \\
& +\frac{\chi}{\kappa}\left(\frac{1}{2} g_{\mu \nu} B^{\alpha} B^{\beta} R_{\alpha \beta}-B_{\mu} B^{\alpha} R_{\alpha \nu}-B_{\nu} B^{\alpha} R_{\alpha \mu}\right. \\
& \left.+\frac{1}{2} \nabla_{\alpha} \nabla_{\mu}\left(B^{\alpha} B_{\nu}\right)+\frac{1}{2} \nabla_{\alpha} \nabla_{\nu}\left(B^{\alpha} B_{\mu}\right)-\frac{1}{2} \nabla^{2}\left(B^{\mu} B_{\nu}\right)-\frac{1}{2} g_{\mu \nu} \nabla_{\alpha} \nabla_{\beta}\left(B^{\alpha} B^{\beta}\right)\right) .
\end{aligned}
$$

In case of fixing the bumblebee field to $B_{\mu}=b_{\mu}$ and subsequently $V=0=V^{\prime}$, then the extended gravitational field equation (2.2), finally reduces to

$$
\begin{aligned}
& R_{\mu \nu}+\kappa b_{\mu \alpha} b_{\nu}^{\alpha}-\frac{\kappa}{4} g_{\mu \nu} b^{\alpha \beta} b_{\alpha \beta}+\chi b_{\mu} b^{\alpha} R_{\alpha \nu}+\chi b_{\nu} b^{\alpha} R_{\alpha \mu}-\frac{\chi}{2} g_{\mu \nu} b^{\alpha} b^{\beta} R_{\alpha \beta}- \\
& \frac{\chi}{2}\left(\nabla_{\alpha} \nabla_{\mu}\left(b^{\alpha} b_{\nu}\right)+\nabla_{\alpha} \nabla_{\nu}\left(b^{\alpha} b_{\mu}\right)-\nabla^{2}\left(b_{\mu} b_{\nu}\right)\right)=0 .
\end{aligned}
$$

By adopting the standard Boyer-Lindquist coordinates, the underlying gravity model admits a Kerrlike black hole solution as [85]

$$
d s^{2}=g_{t t} d t^{2}+g_{r r} d r^{2}+g_{\theta \theta} d \theta^{2}+g_{\phi \phi} d \phi^{2}+2 g_{t \phi} d t d \phi,
$$

where

$$
\begin{aligned}
& g_{t t}=-\left(1-\frac{2 M r}{\varrho^{2}}\right), \quad g_{r r}=\frac{\varrho^{2}}{\Delta_{r}}, \quad g_{\theta \theta}=\varrho^{2}, \quad g_{\phi \phi}=\frac{A \sin ^{2} \theta}{\varrho^{2}} \\
& g_{t \phi}=-\frac{2 M r a \sqrt{\alpha+1} \sin ^{2} \theta}{\varrho^{2}},
\end{aligned}
$$


with

$$
\varrho^{2}=r^{2}+(\alpha+1) a^{2} \cos ^{2} \theta, \quad \Delta_{\alpha}=\frac{r^{2}-2 M r}{\alpha+1}+a^{2}, \quad A=\left(r^{2}+(\alpha+1) a^{2}\right)^{2}-\Delta_{\alpha}(\alpha+1)^{2} a^{2} \sin ^{2} \theta .
$$

In the above solution the spontaneously Lorentz symmetry breaking, addressed by the constant parameter $\alpha$, so that by discarding it then this solution is reduced to the standard Kerr black hole. More exactly, the metric (2.5) denotes a purely radial Lorentz-violating black hole solution with the ADM mass $M$, the angular momentum (per unit mass) $a$ and also a Lorentz-violating parameter $\alpha$. The location of the inner and outer horizons acquire via

$$
r_{\mp}=M \mp \sqrt{M^{2}-a^{2}(\alpha+1)},
$$

which are correspond to the Cushy and event horizons labeled with $r_{c h}$ and $r_{e h}$, respectively. At first glance, one may tell the radius of inner and outer horizons are not distinguishable from their standard counterpart since we can easily absorb the Lorentz-violating parameter $\alpha$ via redefying $a \rightarrow$ $\hat{a}=\sqrt{\alpha+1} a(\alpha>-1)$. Although this seems to be true, it may carry the misleading message that the metric (2.5) is nothing but a standard Kerr metric. However, after applying a such redefinition for $a$, the metric (2.5) re-express as

$$
d s^{2}=-\left(1-\frac{2 M r}{\varrho^{2}}\right) d t^{2}-\frac{4 M r \hat{a} \sin ^{2} \theta}{\varrho^{2}} d t d \varphi+\frac{\varrho^{2}}{\Delta_{r}} d r^{2}+\varrho^{2} d \theta^{2}+\frac{A \sin ^{2} \theta}{\varrho^{2}} d \varphi^{2},
$$

with

$$
\varrho^{2}=r^{2}+\hat{a}^{2} \cos ^{2} \theta, \quad \Delta_{\alpha}=\frac{r^{2}-2 M r+\hat{a}^{2}}{\alpha+1}, \quad A=\left(r^{2}+\hat{a}^{2}\right)^{2}-\left(r^{2}-2 M r+\hat{a}^{2}\right) \hat{a}^{2} \sin ^{2} \theta,
$$

where reveals a slight deviation from the standard Kerr. As a quantity of the underlying background that is expected to appear in the rest of the paper, should be noted to the horizon angular velocity i.e. $\hat{\Omega}_{h}=-\left.\frac{g_{t \phi}}{g_{\phi \phi}}\right|_{r=r_{e h}}=\frac{\hat{a}}{r_{e h}^{2}+\hat{a}^{2}}$. Henceforth, to track the role of the mentioned slight deviation arising from the spontaneously Lorentz symmetry breaking on the black hole superradince, we work with the metric (2.9) in which the angular momentum, labeled by $\hat{a}$.

\section{SUPERRADIANCE SCATTERING OF SCALAR WAVE BY BUMBLEBEE KERR-BLACK HOLE}

The central aim of this paper is probing the trace of the Lorentz-violating parameter $\alpha$ on the superradiance amplification factors which, in essence, address the amount of energy extracted from a Kerr-like background (2.9) due to a massive scalar field scattering. We are looking to do this through an analytical treatment.

\section{A. Equation of motion in Bumblebee Kerr background}

Because of our interest in superradiance scattering of a scalar field $\Phi$ with mass $\mu$, thereby, we have to focus on the solving of the Klein-Gordon equation

$$
\left(\nabla_{\alpha} \nabla^{\alpha}+\mu^{2}\right) \Phi(t, r, \theta, \phi)=0 .
$$


The standard Boyer-Lindquist coordinates $(t, r, \theta, \phi)$ let us to a natural separation of equation (3.1) via following ansats,

$$
\Phi(t, r, \theta, \phi)=F_{\omega l m}(r) S_{\omega l m}(\theta) e^{-i \omega t} e^{-i m \phi}, \quad l \geq 0, \quad-l \leq m \leq l, \quad \omega>0
$$

include the radial function $F_{\omega l m}(r)$ and oblate spheroidal wave function $S_{\omega l m}(\theta)$ in which the indices $l, m$ and $\omega$ respectively represent an integer labelling the angular eigenfunctions, angular quantum number and the positive frequency of the scattering field as measured by an far away observer.

Putting the ansats (3.2) into the partial differential equation (3.1), we deal with two separated ordinary differential equations

$$
\begin{aligned}
& \frac{d}{d r}\left(\Delta_{\alpha} \frac{d F_{\omega l m}(r)}{d r}\right)+\left(\frac{\left(\left(r^{2}+\hat{a}^{2}\right) \omega-\hat{a} m\right)^{2}}{\Delta_{\alpha}}-\left(\mu^{2} r^{2}+l(l+1)+\hat{a}^{2} \omega^{2}-2 m \hat{a} \omega\right)\right) F_{\omega l m}(r)=0, \\
& \sin \theta \frac{d}{d \theta}\left(\sin \theta \frac{d S_{\omega l m}(\theta)}{d \theta}\right)+\left(l(l+1) \sin ^{2} \theta-\left(\left(\hat{a} \omega \sin ^{2} \theta-m\right)^{2}+\hat{a}^{2} \mu^{2} \sin ^{2} \theta \cos ^{2} \theta\right)\right) S_{\omega l m}(\theta)=0 .
\end{aligned}
$$

In direction of our aim, from now on we have to leave the angular equation (3.3b) and concentrate on the radial equation (3.3a). Applying a Regge-Wheeler-like coordinate $r_{*}$ as

$$
r_{*} \equiv \int d r \frac{r^{2}+\hat{a}^{2}}{\Delta_{\alpha}}, \quad\left(r_{*} \rightarrow-\infty \quad \text { at event horizon, } \quad r_{*} \rightarrow \infty \quad \text { at infinity }\right)
$$

along with introducing a new radial function $\mathcal{F}_{\omega l m}\left(r_{*}\right)=\sqrt{r^{2}+\hat{a}^{2}} F_{\omega l m}(r)$, after some calculation one can finally write a Schrödinger-like form of deferential equation

$$
\frac{d^{2} \mathcal{F}_{\omega l m}\left(r_{*}\right)}{d r_{*}^{2}}+U_{\omega l m}(r) \mathcal{F}_{\omega l m}\left(r_{*}\right)=0
$$

with the following scattering potential

$U_{\omega l m}(r)=\left(\omega-\frac{m \hat{a}}{r^{2}+\hat{a}^{2}}\right)^{2}-\frac{\Delta_{\alpha}}{\left(r^{2}+\hat{a}^{2}\right)^{2}}\left(l(l+1)+\hat{a}^{2} \omega^{2}-2 m \hat{a} \omega+\mu^{2} r^{2}\right)+\frac{2 \Delta_{\alpha}\left(\hat{a}^{2}-M r\right)}{\left(r^{2}+\hat{a}^{2}\right)^{3}}-\frac{3 \hat{a}^{2} \Delta_{\alpha}^{2}}{\left(r^{2}+\hat{a}^{2}\right)^{4}}$.

Given the fact that the boundary conditions have a central role in the study of scattering processes, so we have to construct sets of basis modes for the underlying massive scalar field subject to proper boundary conditions at the event horizon and spatially infinity. Concerning the asymptotic treatments of the scattering potential in equation (3.6), we come to constant values,

$$
\lim _{r \rightarrow r_{e h}} U_{\omega l m}(r)=\left(\omega-m \tilde{\Omega}_{h}\right)^{2} \equiv k_{e h}^{2},
$$

and

$$
\lim _{r \rightarrow \infty} U_{\omega l m}(r)=\omega^{2}-\lim _{r \rightarrow \infty} \frac{\mu^{2} r^{2} \Delta_{\alpha}}{\left(r^{2}+\hat{a}^{2}\right)^{2}}=\omega^{2}-\hat{\mu}^{2} \equiv k_{\infty}^{2}, \quad \hat{\mu}=\frac{\mu}{\sqrt{\alpha+1}}
$$

at two extremal points horizon and infinity, respectively. Solving the equation (3.5) asymptotically, the full radial solution take the following form

$$
F_{\omega l m}(r) \rightarrow \begin{cases}\frac{\mathcal{A}_{i n}^{e h} e^{-i k_{e h} r_{*}}}{\sqrt{r_{e h}^{2}+\hat{a}^{2}}} & \text { for } r \rightarrow r_{e h} \\ \mathcal{A}_{i n}^{\infty} \frac{e^{-i k_{\infty} r *}}{r}+\mathcal{A}_{r e f}^{\infty} \frac{e^{i k_{\infty} r *}}{r} & \text { for } r \rightarrow \infty\end{cases}
$$


The indices of the amplitudes $\mathcal{A}$, address the incoming ("in") or reflected ("ref") parts of the scalar wave at the event horizon ("eh") or at infinity ( $\infty$ "). Note that in the above asymptotically solution at the event horizon there was a reflected wave across the surface $r=r_{e h}$ which usually due to the presence of a one-sided membrane as horizon and also well-posed Cauchy problem, it is thrown away. In this point, by facing the Wronskian of the regions near the event horizon $W_{e h}=\left(F_{\omega l m}^{e h} \frac{d F_{\omega l m}^{*} e h}{d r_{*}}-F_{\omega l m}^{* e h} \frac{d F_{\omega l m}^{e h}}{d r_{*}}\right)$ and at infinity $W_{\infty}=\left(F_{\omega l m}^{\infty} \frac{d F_{\omega l m}^{*}}{d r_{*}}-F_{\omega l m}^{*} \frac{d F_{\omega l m}^{\infty}}{d r_{*}}\right)$ together, we finally have

$$
\left|\mathcal{A}_{r e f}^{\infty}\right|^{2}=\left|\mathcal{A}_{i n}^{\infty}\right|^{2}-\frac{k_{e h}}{k_{\infty}}\left|\mathcal{A}_{i n}^{e h}\right|^{2},
$$

where is completely free of the details of the scattering potential in Schrödinger-like differential equation. Above equality openly tells us that in case of $\frac{k_{e h}}{k_{\infty}}<0$ i.e. $\omega<m \hat{\Omega}_{e h}$, then the scalar wave is superradiantly amplified, $\left|\mathcal{A}_{\text {ref }}^{\infty}\right|^{2}>\left|\mathcal{A}_{\text {in }}^{\infty}\right|^{2}$.

\section{B. Computation of scalar superradiance amplification factors $Z_{l m}$}

Because the radial equation (3.3a) is not solvable analytically so we have to resort to some semianalytically methods. One of the most widely used approximate methods is so-called asymptotic matching technique which dates back to seminal work of Starobinsky at early 80's [17]. So, despite the absence of an exact solution for a singularly perturbed differential equation as (3.3a), one still be able to construct an approximation of the solution via asymptotic expansions in relevant extremal points. Based on this method actually one find two approximate solutions each one is valid for part of the range of the independent variable so that finally by their combining together, obtain a trustable single approximate solution. The underlying technique includes two limiting assumptions: $\hat{a} \omega \ll 1$ i.e. $\mathcal{O}(\hat{a} \omega)$ and $M \omega \ll 1$ i.e. $\mathcal{O}(M \omega)$ or $\mathcal{O}(\hat{\mu} M)$ [18]. Former restrict us to low frequency along with slowly rotating regimes for compound system of scalar field and Kerr like black hole. Latter forces us to considering the small black hole so that the relevant Compton wavelength of the massive scalar field is bigger than the size of the black hole. Technically speaking, by applying the asymptotic matching method in a Kerr background, we are deal with two zones: region near the horizon $\left(r-r_{e h} \ll \omega^{-1}\right)$ called the "near-region", and region away from the horizon $\left(r-r_{e h} \gg M\right)$ called the "far-region", outside the event horizon. Given that matching is possible only when the relevant expansions have a domain of overlap so the exact solutions derived for the above two asymptotic regions are matched in an overlapping region where $M \ll r-r_{e h} \ll \omega^{-1}$.

Throughout this subsection, we intend to compute the "amplification factor" of a massive scalar wave scattering off a Bumblebee Kerr black hole through the method discussed above. By this means, we are able to detect the effect of spontaneously Lorentz symmetry breaking addressed by parameter $\alpha$ on the amplification factor $Z_{l m} \equiv \frac{\left|\mathcal{A}_{r e f}^{\infty}\right|^{2}}{\left|\mathcal{A}_{i n}^{\infty}\right|^{2}}-1$, a dimensionless criterion which represents a black hole superradiance, if $Z_{l m}>0$. In what follows, by applying the above discussed semi-analytically technique, we solve the radial equation (3.3a) to deriving $Z_{l m}$.

First, Let's rewrite the radial equation (3.3a) in the form below

$$
\begin{aligned}
& \Delta_{\alpha}^{2} \frac{d^{2} F_{\omega l m}(r)}{d r^{2}}+\Delta_{\alpha} \frac{d \Delta_{\alpha}}{d r} \cdot \frac{d F_{\omega l m}(r)}{d r} \\
& +\left(\left(\left(r^{2}+\hat{a}^{2}\right) \omega-\hat{a} m\right)^{2}-\Delta_{\alpha}\left(\mu^{2} r^{2}+l(l+1)+\hat{a}^{2} \omega^{2}-2 m \hat{a} \omega\right)\right) F_{\omega l m}(r)=0
\end{aligned}
$$


Our strategy for solving the above differential equation consists of three parts: i) deriving the nearregion solution, ii) deriving the far-region solution and iii) matching solutions to having a single solution. First of all, the equation (3.11) after applying the change of variable $x=\frac{r-r_{h}}{r_{h}-r_{c}}$ along with plugging $\triangle_{\alpha} \frac{d}{d r}=\frac{\left(r_{e h}-r_{c h}\right)}{\alpha+1} x(x+1) \frac{d}{d x}$ and also using approximation $\hat{a} \omega \ll 1$, reads as

$$
\begin{aligned}
& \frac{x^{2}(x+1)^{2}}{(\alpha+1)^{2}} \frac{\mathrm{d}^{2} F_{\omega l m}(x)}{\mathrm{d} x^{2}}+\frac{x(x+1)(2 x+1)}{(\alpha+1)^{2}} \frac{\mathrm{d} F_{\omega l m}(x)}{\mathrm{d} x} \\
& +\left(A x^{4}+B^{2}-\frac{l(l+1)}{\alpha+1} x(x+1)-\frac{\hat{\mu}^{2} A^{2}}{\omega^{2}} x^{3}(x+1)-\hat{\mu}^{2} r_{e h}^{2} x(x+1)-\frac{2 \hat{\mu}^{2} r_{e h}^{2} A}{\omega} x^{2}(x+1)\right) F_{\omega l m}(x)=0,
\end{aligned}
$$

where $A=\left(r_{e h}-r_{c h}\right) \omega$ and $B=\frac{(\omega-m \hat{\Omega})}{r_{e h}-r_{c h}} r_{e h}^{2}$. By focusing on part i, the equation (3.12) due to two applicable approximations in regions close to the horizon i.e. $A x \ll$ and $\hat{\mu}^{2} r_{e h}^{2} \ll 1$, reduces to

$$
x^{2}(x+1)^{2} \frac{\mathrm{d}^{2} F_{\omega l m}(r)}{\mathrm{d} x^{2}}+x(x+1)(2 x+1) \frac{\mathrm{d} F_{\omega l m}(r)}{\mathrm{d} x}+\left((\alpha+1)^{2} B^{2}-l(l+1)(\alpha+1) x(x+1)\right) F_{\omega l m}(r)=0 .
$$

Note that the second approximation $\left(\hat{\mu}^{2} r_{e h}^{2} \ll 1\right)$ comes from Compton wavelength approximation as one of central assumptions in asymptotic matching technique. The general solution of equation (3.13) satisfying the ingoing boundary condition, written in terms of Legendre function of the first kind $P_{\lambda}^{\nu}(y)$

$$
F_{\omega l m}(x)=c P_{\frac{\sqrt{1+4(\alpha+1) l(l+1)}-1}{2}}^{2 i(\alpha+1) B}(1+2 x),
$$

where using following relation

$$
P_{\lambda}^{\nu}(y)=\frac{1}{\Gamma(1-\nu)}\left(\frac{1+y}{1-y}\right)_{2}^{\nu / 2} F_{1}\left(-\lambda, \lambda+1 ; 1-\nu ; \frac{1-y}{2}\right),
$$

it finally re-express in terms of ordinary hypergeometric functions ${ }_{2} F_{1}(a, b ; c ; y)$

$$
F_{\omega l m}(x)=c\left(\frac{x}{x+1}\right)^{-i(\alpha+1) B}{ }_{2} F_{1}\left(\frac{1-\sqrt{1+4(\alpha+1) l(l+1)}}{2}, \frac{1+\sqrt{1+4(\alpha+1) l(l+1)}}{2} ; 1-2 i(\alpha+1) B ;-x\right) .
$$

Matching of solutions requires us to looking for the manner of above solution at large $x$ i.e. ${ }^{7}$

$$
\begin{aligned}
F_{\text {near-large } \mathrm{x}} \sim c & \left(\frac{\Gamma(\sqrt{1+4(\alpha+1) l(l+1)}) \Gamma(1-2 i(\alpha+1) B)}{\Gamma\left(\frac{1+\sqrt{1+4(\alpha+1) l(l+1)}}{2}-2 i(\alpha+1) B\right) \Gamma\left(\frac{1+\sqrt{1+4(\alpha+1) l(l+1)}}{2}\right)} x^{\frac{\sqrt{1+4(\alpha+1) l(l+1)}-1}{2}}+\right. \\
& \left.\frac{\Gamma(-\sqrt{1+4(\alpha+1) l(l+1)}) \Gamma(1-2 i(\alpha+1) B)}{\Gamma\left(\frac{1-\sqrt{1+4(\alpha+1) l(l+1)}}{2}\right) \Gamma\left(\frac{1-\sqrt{1+4(\alpha+1) l(l+1)}}{2}-2 i(\alpha+1) B\right)} x^{-\frac{\sqrt{1+4(\alpha+1) l(l+1)}+1}{2}}\right) .
\end{aligned}
$$

\footnotetext{
7 To obtain equation (3.17), used from asymptotic behaviour of the hypergeometric function $\lim _{x \rightarrow \infty}{ }_{2} F_{1}(a, b ; c ;-y)=$ $\frac{\Gamma(b-a) \Gamma(c)}{\Gamma(c-a) \Gamma(b)} y^{-a}+\frac{\Gamma(a-b) \Gamma(c)}{\Gamma(c-b) \Gamma(a)} y^{-b}$
} 
By going to part ii of strategy in order to deriv the far-region solution, equation (3.11) reads as

$$
\frac{\mathrm{d}^{2} F_{\omega l m}(x)}{\mathrm{d} x^{2}}+\frac{2}{x} \frac{\mathrm{d} F_{\omega l m}(x)}{\mathrm{d} x}+\left(k^{2}-\frac{l(l+1)(\alpha+1)}{x^{2}}\right) F_{\omega l m}(x)=0,
$$

where $k \equiv \frac{A}{\omega} \sqrt{\omega^{2}-\hat{\mu}^{2}}$. Note that here we used from approximations $x+1 \approx x$ and $\hat{\mu}^{2} r_{e h}^{2} \ll 1$. For the equation (3.18), we have following general solution

$$
\begin{array}{r}
F_{\omega l m, \text { far }}=e^{-i k x}\left(d_{1} x^{\frac{\sqrt{1+4(\alpha+1) l(l+1)}-1}{2}} U\left(\frac{1+\sqrt{1+4(\alpha+1) l(l+1)}}{2}, 1+\sqrt{1+4(\alpha+1) l(l+1)}, 2 i k x\right)+\right. \\
\left.d_{2} x^{-\frac{\sqrt{1+4(\alpha+1) l(l+1)}-1}{2}} U\left(\frac{1-\sqrt{1+4(\alpha+1) l(l+1)}}{2}, 1-\sqrt{1+4(\alpha+1) l(l+1)}, 2 i k x\right)\right),
\end{array}
$$

where $U(a, b, y)$ refers to the first Kummer function. Matching of solutions requires us to looking for the manner of above solution at small $x$ i.e. ${ }^{8}$

$$
F_{\omega l m, \text { far-small x }} \sim d_{1} x^{\frac{\sqrt{1+4(\alpha+1) l(l+1)}-1}{2}}+d_{2} x^{-\frac{1+\sqrt{1+4(\alpha+1) l(l+1)}}{2}} .
$$

Now we are in suitable point to apply part iii of strategy i.e. matching above derived two asymptotic solutions to calculate the scalar wave fluxes at infinity. In this way, we will have the relevant expression for the amplification factor. At first step, by facing equations (3.17) and (3.20) together we acquire

$$
\begin{aligned}
& d_{1}=c \frac{\Gamma(\sqrt{1+4(\alpha+1) l(l+1)}) \Gamma(1-2 i(\alpha+1) B)}{\left.\Gamma\left(\frac{1+\sqrt{1+4(\alpha+1) l(l+1)}}{2}\right)-2 i(\alpha+1) B\right) \Gamma\left(\frac{1+\sqrt{1+4(\alpha+1) l(l+1)}}{2}\right)}, \\
& d_{2}=c \frac{\Gamma(-\sqrt{1+4(\alpha+1) l(l+1)}) \Gamma(1-2 i(\alpha+1) B)}{\left.\Gamma\left(\frac{1-\sqrt{1+4(\alpha+1) l(l+1)}}{2}\right)-2 i(\alpha+1) B\right) \Gamma\left(\frac{1-\sqrt{1+4(\alpha+1) l(l+1)}}{2}\right)} .
\end{aligned}
$$

Now we need to connect the coefficients $d_{1}$ and $d_{2}$ with coefficients $\mathcal{A}_{i n}^{\infty}$ and $\mathcal{A}_{r e f}^{\infty}$ in the radial solution (3.9). To do this, we expand the far region solution (3.19) around infinity as

$$
\begin{gathered}
d_{1} \frac{\Gamma(1+\sqrt{1+4(\alpha+1) l(l+1)})}{\Gamma\left(\frac{1+\sqrt{1+4(\alpha+1) l(l+1)}}{2}\right)} k^{-\frac{1+\sqrt{1+4(\alpha+1) l(l+1)}}{2}}\left((-2 i)^{-\frac{1+\sqrt{1+4(\alpha+1) l(l+1)}}{2}} \frac{e^{-i k x}}{x}+(2 i)^{-\frac{1+\sqrt{1+4(\alpha+1) l(l+1)}}{2}} \frac{e^{i k x}}{x}\right)+ \\
d_{2} \frac{\Gamma(1-\sqrt{1+4(\alpha+1) l(l+1)})}{\frac{1-\sqrt{1+4(\alpha+1) l(l+1)}}{2}} k^{\frac{\sqrt{1+4(\alpha+1) l(l+1)}-1}{2}}\left((-2 i)^{\frac{\sqrt{1+4(\alpha+1) l(l+1)}-1}{2}} \frac{e^{-i k x}}{x}+(2 i)^{\frac{\sqrt{1+4(\alpha+1) l(l+1)}-1}{2}} \frac{e^{i k x}}{x}\right) .
\end{gathered}
$$

Using the approximations $\frac{1}{x} \sim \frac{A}{\omega} \cdot \frac{1}{r}, \quad e^{ \pm i k x} \sim e^{ \pm i \sqrt{\omega^{2}-\tilde{\mu}^{2}} r}$ and then matching the solution (3.22) with the radial solution (3.9)

$$
F_{\infty}(r) \sim \mathcal{A}_{i n}^{\infty} \frac{e^{-i \sqrt{\omega^{2}-\tilde{\mu}^{2}} r^{*}}}{r}+\mathcal{A}_{r e f}^{\infty} \frac{e^{i \sqrt{\omega^{2}-\tilde{\mu}^{2}} r^{*}}}{r}, \quad \text { for } \quad r \rightarrow \infty,
$$

\footnotetext{
${ }^{8}$ Here used from the Taylor expansion $\lim _{y \rightarrow 0} U(a, b, y) \approx \frac{\Gamma(1-b)}{\Gamma(1+a-b)}+\ldots$
} 
we have

$$
\begin{aligned}
& \mathcal{A}_{\text {in }}^{\infty}=\frac{A}{\omega}\left(d_{1}(-2 i)^{-\frac{1+\sqrt{1+4(\alpha+1) l(l+1)}}{2}} \frac{\Gamma(1+\sqrt{1+4(\alpha+1) l(l+1)})}{\Gamma\left(\frac{1+\sqrt{1+4(\alpha+1) l(l+1)}}{2}\right)} k^{-\frac{1+\sqrt{1+4(\alpha+1) l(l+1)}}{2}}+\right. \\
& \left.d_{2}(-2 i)^{\frac{\sqrt{1+4(\alpha+1) l(l+1)}-1}{2}} \frac{\Gamma(1-\sqrt{1+4(\alpha+1) l(l+1)})}{\Gamma\left(\frac{1-\sqrt{1+4(\alpha+1) l(l+1)}}{2}\right)} k^{\frac{\sqrt{1+4(\alpha+1) l(l+1)}-1}{2}}\right),
\end{aligned}
$$

and

$$
\begin{aligned}
& \mathcal{A}_{\text {ref }}^{\infty}=\frac{A}{\omega}\left(d_{1}(2 i)^{-\frac{1+\sqrt{1+4(\alpha+1) l(l+1)}}{2}} \frac{\Gamma(1+\sqrt{1+4(\alpha+1) l(l+1)})}{\Gamma\left(\frac{1+\sqrt{1+4(\alpha+1) l(l+1)}}{2}\right)} k^{-\frac{1+\sqrt{1+4(\alpha+1) l(l+1)}}{2}}+\right. \\
& \left.d_{2}(2 i)^{\frac{\sqrt{1+4(\alpha+1) l(l+1)}-1}{2}} \frac{\Gamma(1-\sqrt{1+4(\alpha+1) l(l+1)})}{\Gamma\left(\frac{1-\sqrt{1+4(\alpha+1) l(l+1)}}{2}\right)} k^{\frac{\sqrt{1+4(\alpha+1) l(l+1)}-1}{2}}\right) .
\end{aligned}
$$

As the last step, by adding the expressions obtained in (3.21) for $d_{1}$ and $d_{2}$, the final form of $\mathcal{A}_{\text {ref }}^{\infty}$ and $\mathcal{A}_{\text {in }}^{\infty}$, written as

$$
\begin{aligned}
& \mathcal{A}_{i n}^{\infty}=\frac{c(-2 i)^{-\frac{1+\sqrt{1+4(\alpha+1) l(l+1)}}{2}}}{\sqrt{\omega^{2}-\tilde{\mu}^{2}}} \cdot \frac{\Gamma(\sqrt{1+4(\alpha+1) l(l+1)}) \Gamma(1+\sqrt{1+4(\alpha+1) l(l+1)})}{\Gamma\left(\frac{1+\sqrt{1+4(\alpha+1) l(l+1)}}{2}-2 i(\alpha+1) B\right)\left(\Gamma\left(\frac{1+\sqrt{1+4(\alpha+1) l(l+1)}}{2}\right)\right)^{2}}(8.26) \\
& \Gamma(1-2 i(\alpha+1) B) k^{\frac{1-\sqrt{1+4(\alpha+1) l(l+1)}}{2}}+\frac{c(-2 i)^{\frac{\sqrt{1+4(\alpha+1) l(l+1)}-1}{2}}}{\sqrt{\omega^{2}-\hat{\mu}^{2}}} \times \\
& \left.\frac{\Gamma(1-\sqrt{1+4(\alpha+1) l(l+1)}) \Gamma(-\sqrt{1+4(\alpha+1) l(l+1)})}{\left(\Gamma\left(\frac{1-\sqrt{1+4(\alpha+1) l(l+1)}}{2}\right)\right)^{2} \Gamma\left(\frac{1-\sqrt{1+4(\alpha+1) l(l+1)}}{2}-2 i(\alpha+1) B\right)} \Gamma i(\alpha+1) B\right) k^{\frac{1+\sqrt{1+4(\alpha+1) l(l+1)}}{2}},
\end{aligned}
$$

and

$$
\begin{aligned}
& \mathcal{A}_{r e f}^{\infty}=\frac{c(2 i)^{-\frac{1+\sqrt{1+4(\alpha+1) l(l+1)}}{2}}}{\sqrt{\omega^{2}-\hat{\mu}^{2}}} \cdot \frac{\Gamma(\sqrt{1+4(\alpha+1) l(l+1)}) \Gamma(1+\sqrt{1+4(\alpha+1) l(l+1)})}{\Gamma\left(\frac{1+\sqrt{1+4(\alpha+1) l(l+1)}}{2}-2 i(\alpha+1) B\right)\left(\Gamma\left(\frac{1+\sqrt{1+4(\alpha+1) l(l+1)}}{2}\right)\right)^{2}}(3.27) \\
& \Gamma(1-2 i(\alpha+1) B) k^{\frac{1-\sqrt{1+4(\alpha+1) l(l+1)}}{2}}+\frac{c(2 i)^{\frac{\sqrt{1+4(\alpha+1) l(l+1)}-1}{2}}}{\sqrt{\omega^{2}-\hat{\mu}^{2}}} \times \\
& \frac{\Gamma(1-\sqrt{1+4(\alpha+1) l(l+1)}) \Gamma(-\sqrt{1+4(\alpha+1) l(l+1)})}{\left(\Gamma\left(\frac{1-\sqrt{1+4(\alpha+1) l(l+1)}}{2}\right)\right)^{2} \Gamma\left(\frac{1-\sqrt{1+4(\alpha+1) l(l+1)}}{2}-2 i(\alpha+1) B\right)} \Gamma(1-2 i(\alpha+1) B) k^{\frac{1+\sqrt{1+4(\alpha+1) l(l+1)}}{2}} .
\end{aligned}
$$

respectively. Now one able to the calculation of the amplification factor

$$
Z_{l m} \equiv \frac{\left|\mathcal{A}_{r e f}^{\infty}\right|^{2}}{\left|\mathcal{A}_{\text {in }}^{\infty}\right|^{2}}-1,
$$

via equations (3.26) and (3.27). 


\section{Results}

First, we release the results derived above for the leading multipoles $(l=1,2)$ of the massive scalar wave scattered off a Kerr black hole with the ratio angular momentum to mass $\hat{a} / M=0.9$, see Figs. (1) and (2). Indeed, if $Z>0$ it addresses a gain factor assigned to scalar wave mode means happening the superradiance phenomena in the underlying background while $Z<0$ denote a loss factor means the non-occurrence of superradiance.

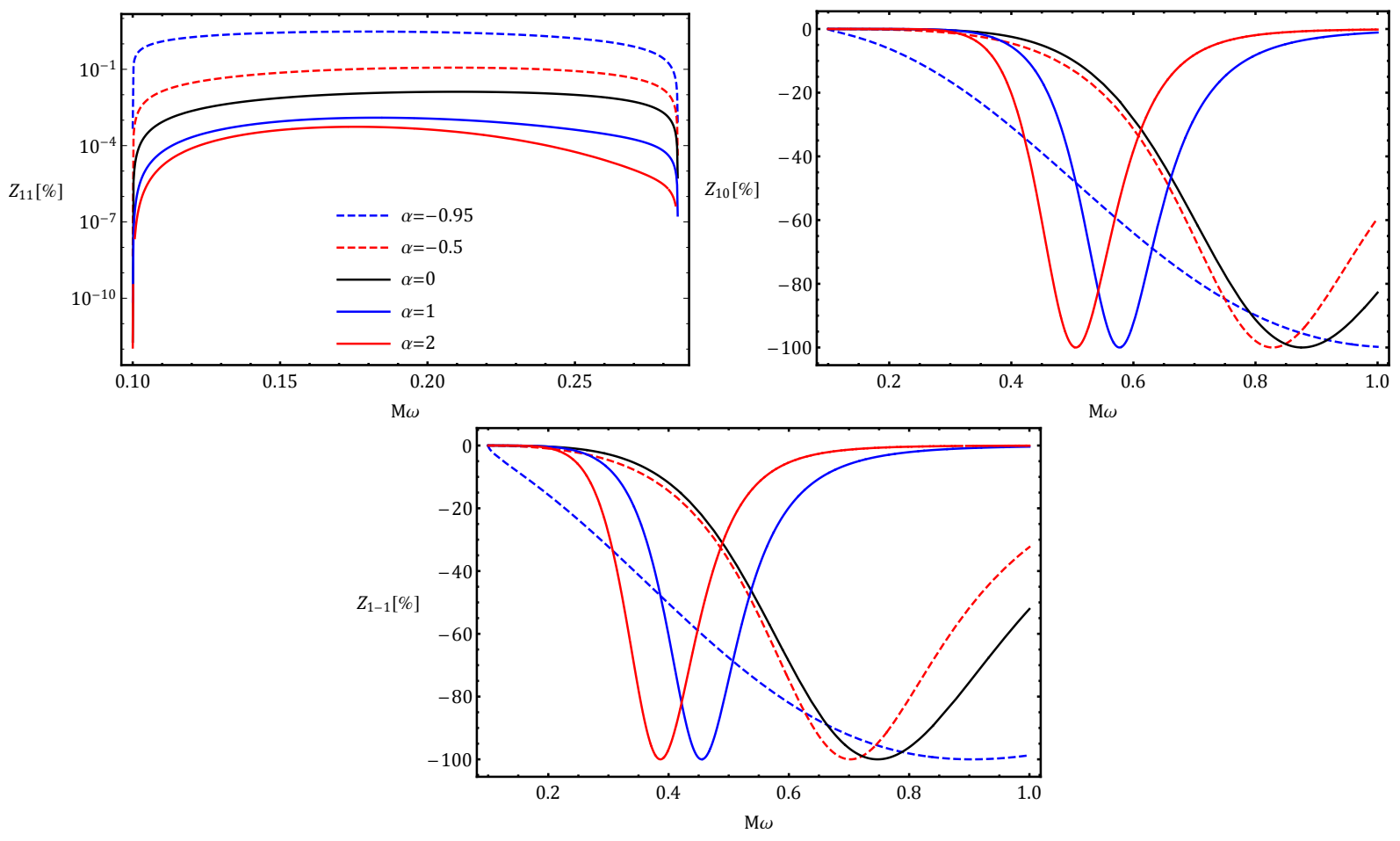

FIG. 1: Analytical results obtained from (3.28) for the amplification factor of the massive scalar wave with the multipoles $(l, m)=(1,1),(1,0),(1,-1)$ and mass $\hat{\mu}=0.1$ scattered off the bumblebee Kerr like background with $\hat{a} / M=0.9$ and different values of Lorentz violating parameter $\alpha$.

Concerning these two figures, we can divide our analysis to two cases non-positive $m \leq 0$ and positive $m>0$. In similar to the standard Kerr black hole, for the case $m \leq 0$ the massive scalar field includes the non-superradiant modes since we deal with $Z \leq 0$ within the relevant frequency range. However, in the presence of Lorentz violating parameter $\alpha$ we see more wave energy loss relative to the standard case within the frequency regions $M \omega \ll 1$ (as our study validity range). Actually, the presence of $\alpha$ in the background seems to accelerate the process of energy loss in the non-superradiant modes. However, by going to case $m>0$, superradiance phenomena will occurs with two distinguishable feedbacks from the Lorentz violating parameter $\alpha$. The plots related to case $m>0$ in the Figs. (1) and (2), clear-cut tell us that $\alpha<0$ results in enhanced superradiance while $\alpha>0$, weakens it. This may seems more interesting if we confront it with the result released in [85] about relation between the Lorentz violating parameter $\alpha$ and the size of the shadow. There, the results indicate that the shadow's size of the Kerr-like black hole at hand, increases, and decreases compared to the standard case respectively for $\alpha<0$ and $\alpha>0$. Due to this comparison, this idea is prone to emerge that the observation of a deviation in the size of 

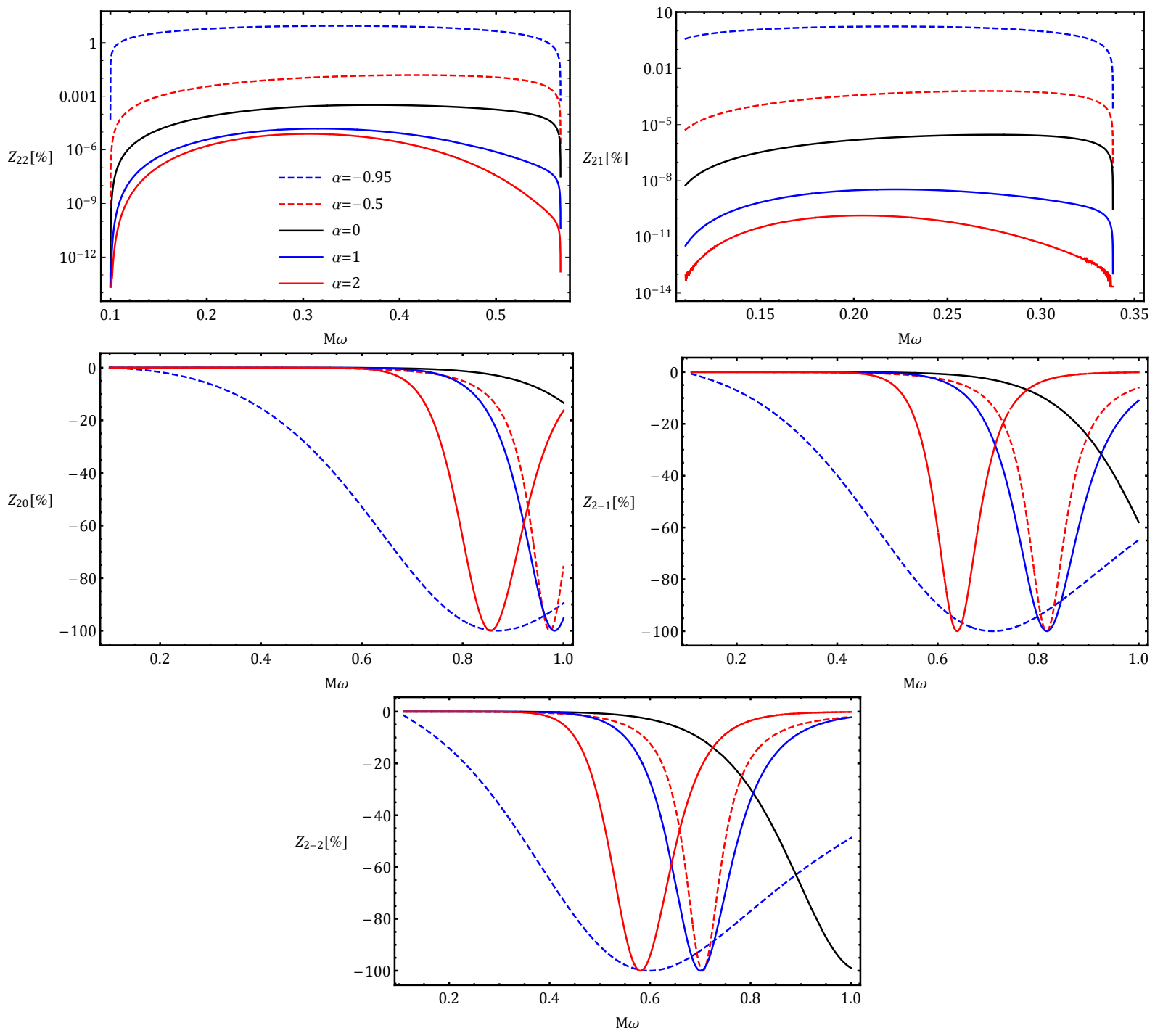

FIG. 2: Analytical results obtained from (3.28) for the amplification factor of the massive scalar wave with the multipoles $(l, m)=(2,2),(2,1),(2,0),(2,-1),(2,-2)$ and mass $\hat{\mu}=0.1$ scattered off the bumblebee Kerr like background with $\hat{a} / M=0.9$ and different values of Lorentz violating parameter $\alpha$.

the shadow in the direction of the increase may be indirect evidence of happening the superradiance phenomenon.

One may want to search for the relation between the black hole's spin $\hat{a}$ and Lorentz violating parameter $\alpha$ with the superradiance amplification factor. In this direction, by choosing two optional values for negative and positive Lorentz violating parameter $\alpha$, we release the superradiance amplification factors $Z_{11}$ and $Z_{22}$ in Fig. (3), for some values of black hole's spin $\hat{a}$. Generally speaking, for both cases $\alpha<0$ and $\alpha>0$ we observe that the superradiance frequency range as well as the their amplification factors become wider and stronger, as the black hole's spin gets bigger. It could contain the message that as the Kerr black hole gets closer to the extremal case $\hat{a}=M$, the effects arising from Lorentz symmetry breaking on the superradiance becomes more noticeable, too. 

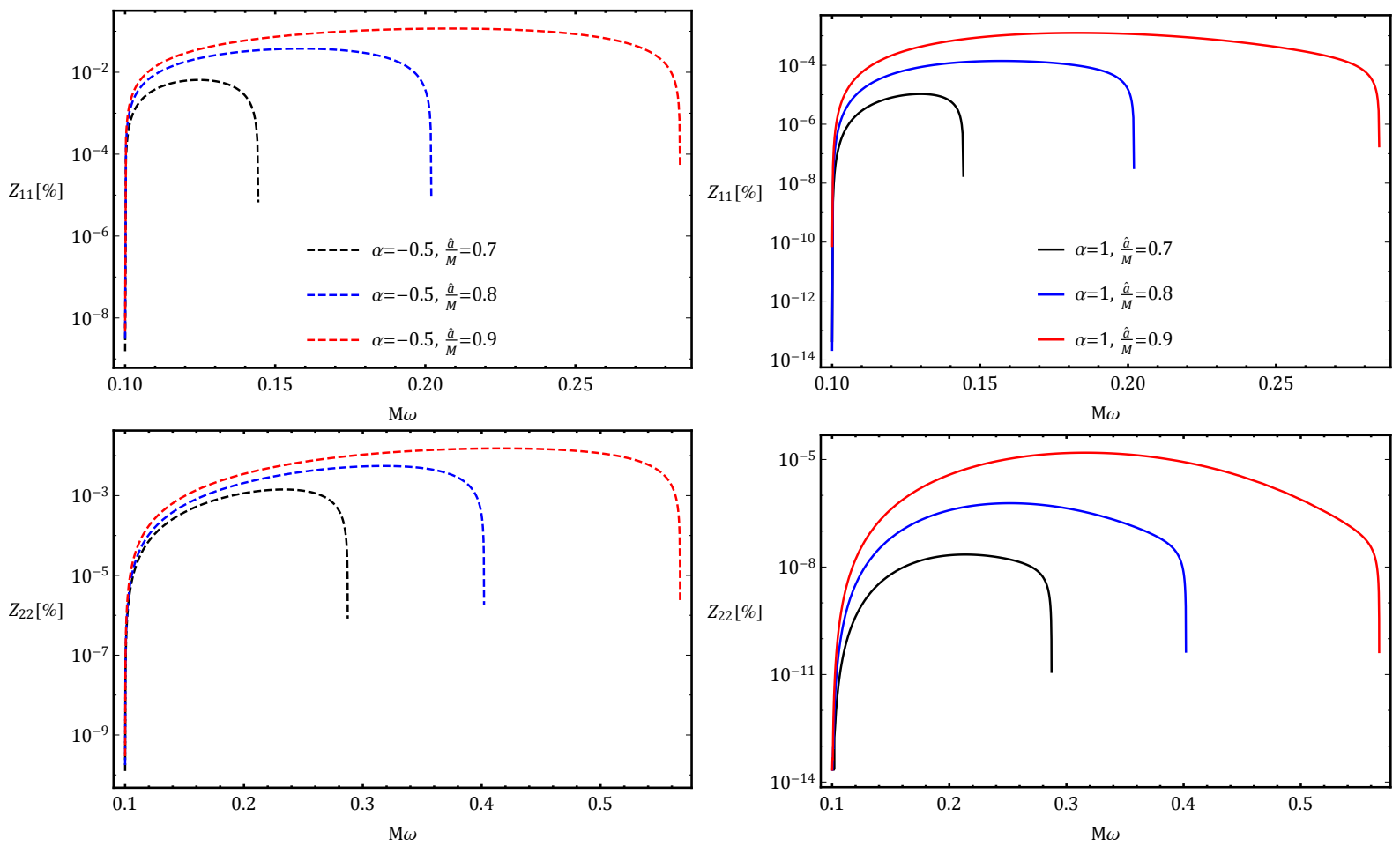

FIG. 3: Amplification factors $Z_{11}$ and $Z_{22}$ for two optional values of $\alpha: \alpha=-0.5$ (left panels in top and bottom rows) and $\alpha=1$ (right panels in top and bottom rows) with different values of $\hat{a} / M=0.7,0.8,0.9$.

\section{SUPERRADIANT INSTABILITY WITH LORENTZ-VIOLATING PARAMETER}

Above, we have discussed on the superradiance amplification of a bosonic field such as massive scalar field due to scattering of a bumblebee-Kerr black hole. Given the fact that superradiance scattering does not necessarily mean instability so here we extend it within a related issue knows as "black hole bomb" for seeing the role of Lorentz-violating parameter on superradiant instability. Technically speaking, the instability of the composite system of Kerr background and massive scalar perturbations may be due to the massive modes which are captured inside the effective potential well outside the black hole. These massive modes, in essence, behave like a mirror so that by returning the reflection waves toward black hole and subsequently their amplifying by creating bounce back and forth moves, give rise to an superradiantly instability known as black hole bomb. Overall, to trigger this instability, the existence of a trapping potential well outside the black hole is crucial in addition to the existence of an ergoregion, to happening the superradiant amplification. In the following, we will investigate this for a Kerr-like background include Lorentz symmetry breaking characterized by constant parameter $\alpha$ which is subjecting to massive scalar perturbation.

Beginning the radial Klein-Gordon equation (3.3a), we have

$$
\Delta_{\alpha} \frac{d}{d r}\left(\Delta_{\alpha} \frac{d F_{\omega l m}}{d r}\right)+\xi F_{\omega l m}=0
$$


where in the slowly rotating regime $(\hat{a} \omega \ll 1)$

$$
\xi \equiv\left(\left(r^{2}+\hat{a}^{2}\right) \omega-m \hat{a}\right)^{2}+\Delta_{\alpha}\left(2 m \hat{a} \omega-l(l+1)-\mu^{2}\left(r^{2}+\hat{a}^{2}\right)\right) .
$$

Demanding the black hole bomb mechanism, we should have the following solutions for the radial equation (4.1)

$$
F_{\omega l m} \sim\left\{\begin{array}{lll}
e^{-i(\omega-m \hat{\Omega}) r_{*}} & \text { as } r \rightarrow r_{e h} \quad\left(r_{*} \rightarrow-\infty\right) \\
\frac{e^{-\sqrt{\mu^{2}-\omega^{2}} r_{*}}}{r} & \text { as } r \rightarrow \infty \quad\left(r_{*} \rightarrow \infty\right)
\end{array}\right.
$$

The solutions above, are represent this physical boundary conditions that the scalar wave at the black hole horizon is purely ingoing while at spatial infinity it is a decaying exponentially (bounded) solution, provided that $\omega^{2}<\mu^{2}$.

By regarding a new radial function as follows

$$
\psi_{\omega l m} \equiv \sqrt{\Delta_{\alpha}} F_{\omega l m}
$$

in the radial equation (4.1), one come to

$$
\left(\frac{d^{2}}{d r^{2}}+\omega^{2}-V\right) \psi_{\omega l m}=0
$$

with

$$
\omega^{2}-V=\frac{\xi+M^{2}-\hat{a}^{2}}{\Delta_{\alpha}^{2}}
$$

where is indeed Regge-Wheel equation. With a straightforward calculation, one can show that the asymptotic form of the effective potential $V$, by discarding terms $\mathcal{O}\left(1 / r^{2}\right)$ is in the form below

$$
V(r)=\mu^{2}-\frac{4 M \omega^{2}}{r}+(\alpha+1) \frac{2 M \mu^{2}}{r} .
$$

To releasing a trapping well by above effective potential it is necessary that its asymptotic derivative be positive i.e $V^{\prime} \rightarrow 0^{+}$as $r \rightarrow \infty$ [33]. As a result, one acquire the following modified regime

$$
\frac{\alpha+1}{2} \mu^{2}<\omega^{2}<\mu^{2}
$$

in which the bound states may capture. It is very obvious that the above regime is well-defined provided that $\alpha>-1$. We have shown before that the superradiance condition for amplification of the scattered waves from underlying background is $\omega<m \hat{\Omega}$ where in case of facing with modified instability regime (4.8), one finally realizes following restricted regime

$$
\sqrt{\frac{\alpha+1}{2}} \mu<\omega<m \hat{\Omega}
$$

in which the integrated system of Kerr bumblebee black hole and massive scalar field, may experience a superradiant instability known as black hole bomb. Namely, the dynamics of the massive scalar 

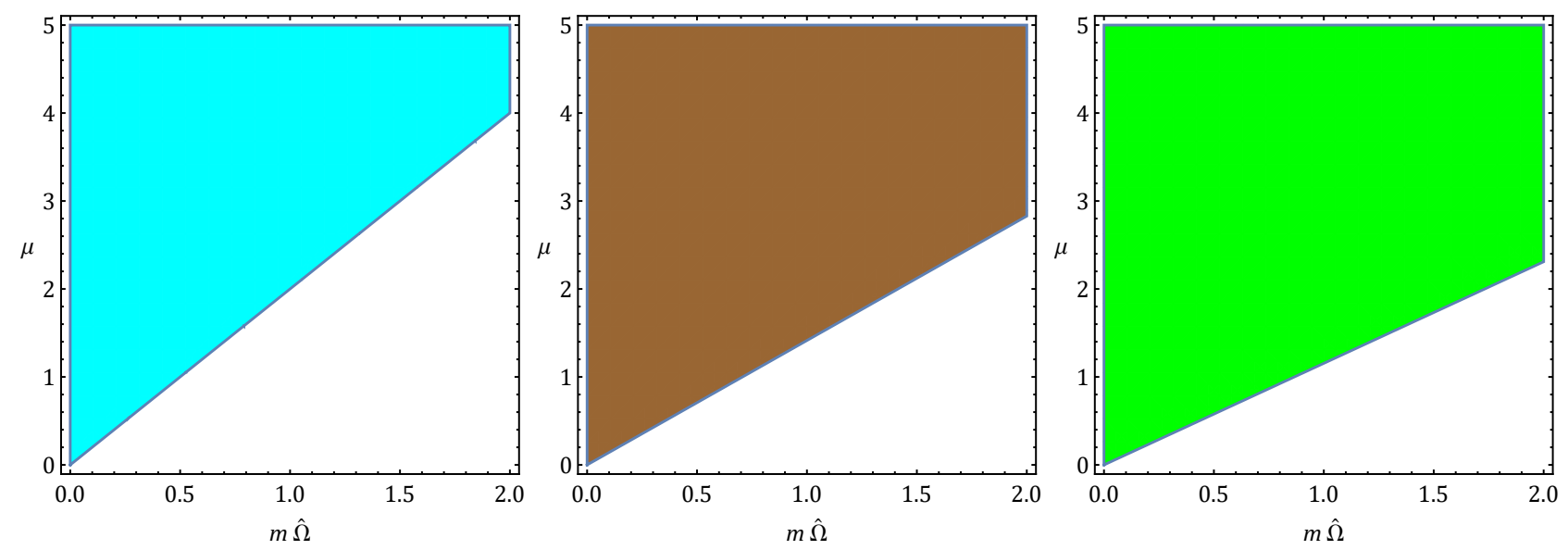

FIG. 4: The parameter space $(m \hat{\Omega}, \mu)$ of massive scalar field in the background of bumblebee-Kerr spacetime include Lorentz violating parameter $\alpha$ with different values:-0.5, 0, 0.5 from left to right, respectively. Colored and non-colored areas respectively represent regions with stable and unstable dynamics for the massive scalar field.

field in confronting with a Lorentz symmetry violating background, is expected to remain stable in the complementary regime $\mu \geq \sqrt{\frac{2}{\alpha+1}} m \hat{\Omega}$. As can be seen from Fig. (4), by going the value of $\alpha$ from negative to positive ones within the allowed range $\alpha>-1$, then the superradiant instability regions become smaller. In other words, as can be seen, the massive scalar filed within a Kerr like background with the positive values of $\alpha$ has a lower chance of causing instability relative to their negative counterparts. This is interesting in the sense that in case of $\alpha<0$ as the massive scalar wave superradiance enhances in compared to its standard counterpart, it also has a better chance of making instability, and vice versa for $\alpha>0$. However, it is important to remember that there is no requirement that in case of larger/smaller energy extraction from background we necessarily deal with a stronger/weaker superradiant instability ${ }^{9}$.

Recently in [92] by employing a numerical method claimed that the superradiant instability due to competing effects arose from the mass losing and spin-down can lead to a decrease and an increase of the shadow radius, respectively. So, dependent on dominating each of these two effects, then it can be said that the superradiant instability decreases or increases the size of the shadow, respectively. Besides, as before mentioned, we know from [85] that moving from $\alpha<0$ to $\alpha>0$, leads to a decrease of shadow radius. With these descriptions, it seems that for $\alpha<0$, the effect of spin-down is dominates on the mass loss while for $\alpha>0$, its opposite happens.

\section{CONCLUSION}

In this paper, we have studied the superradiance phenomenon and its related instability for a spinning-like black hole in the Einstein-bumblebee modified gravity. The key property of such a theory

\footnotetext{
${ }^{9}$ A similar situation has already been seen in a composite system of charged massive scalar field and RN black hole so that in the regime $Q \leq 2 \sqrt{2} / 3 M$, the black hole experiences superradiance without instability, see [91] for more details.
} 
of gravity actually is the presence of a vector field known as bumblebee field with non-zero vacuum expectation value, representing spontaneously breaking of Lorentz symmetry. With the ask of how the spontaneous Lorentz symmetry breaking affects the superradiance scattering and corresponding instability, we have considered a composited system of the massive scalar perturbation and a small rotating black hole admitted by the theory of gravity at hand. By investigating the low-frequency limit through employing asymptotic matching method, we analytical shown that the massive scalar wave amplifies with the Lorentz breaking constant $\alpha<0$, and weakens with $\alpha>0$. Next, from the analytical study of superradiant instability via addressing the issue of the black hole bomb, we found that the Lorentz breaking constant affects the instability regime so that the background with $\alpha<0$ has more chance of unstable dynamics for the scalar field while with $\alpha>0$ less. As a result, the Lorentz breaking constant affects identically the superradiance scattering and relevant instability. Finally, with the integration of our results with the discussions released in Refs. [85] and [92], we have argued that in the superradiant scattering with $\alpha<0$ and $\alpha>0$, respectively spin-down and mass loss of black hole (as two competing influences at play) separately become dominate. By imposing observational constraints on the Lorentz breaking constant $\alpha$ in the future, it may be ruled out one of the two possibilities above.

Acknowledgments: I would like to thank Carlos Herdeiro for fruitful comments and discussions on the manuscript. I thank Sunny Vagnozzi for the detailed discussions on bumblebee gravity. I also need to appreciate Soroush Shakeri for his help in final editing the manuscript.

[1] B. P. Abbott et al. [LIGO Scientific and Virgo Collaborations], Phys. Rev. Lett. 116 (2016) no.6, 061102 [arXiv:1602.03837 [gr-qc]].

[2] B. P. Abbott et al. [LIGO Scientific and Virgo Collaborations], Phys. Rev. X 6 (2016) no.4, 041015 Erratum: [Phys. Rev. X 8 (2018) no.3, 039903] [arXiv:1606.04856 [gr-qc]].

[3] B. P. Abbott et al. [LIGO Scientific and Virgo Collaborations], Phys. Rev. X 9 (2019) no.3, 031040 [arXiv:1811.12907 [astro-ph.HE]].

[4] K. Akiyama et al. [Event Horizon Telescope Collaboration], Astrophys. J. 875 (2019) no.1, L1 [arXiv:1906.11238 [astro-ph.GA]].

[5] K. Akiyama et al. [Event Horizon Telescope Collaboration], Astrophys. J. 875 (2019) no.1, L6 [arXiv:1906.11243 [astro-ph.GA]].

[6] Th. Rivinius, D. Baade, P. Hadrava, M. Heida, R. Klement, arXiv:2005.02541 [astro-ph.SR]

[7] M. Y. Piotrovich, S. V. Krasnikov, S. D. Buliga and T. M. Natsvlishvili, arXiv:2008.09411 [astro-ph.HE].

[8] B. M. Peterson and A. Wandel, Astrophys. J. 540 (2000) L13 [astro-ph/0007147].

[9] R. Emami and A. Loeb, JCAP 2002 (2020) 021 [arXiv:1903.02578 [astro-ph.HE]].

[10] Ya. B. Zeldovich, Pisma Zh. Eksp. Teor. Fiz. 14, 270 (1971) [JETP Lett. 14, 180 (1971)]

[11] Ya. B. Zeldovich, PismaZh. Eksp. Teor. Fiz.62, 2076 (1972) [Sov. Phys. JETP 35, 1085 (1972)].

[12] W. H. Press, and S. A. Teukolsky, Nature (London) 238, 211 (1972).

[13] J. M. Bardeen, W. H. Press, and S. A. Teukolsky, Astrophys. J. 178, 347 (1972).

[14] C. W. Misner, Bull. Am. Phys. Soc. 17, 472(1972).

[15] A. A. Starobinsky, Zh. Eksp. Teor. Fiz. 64, 48 (1973) [Sov. Phys. JETP 37, 28 ( 1973)]

[16] A. A. Starobinsky and S. M. Churilov, Zh. Eksp. Teor. Fiz. 65, 3 (1973) [Sov. Phys. JETP 38, 1 (1973)].

[17] A. A. Starobinsky, Sov. Phys. JETP 37 (1973) no.1, 28 [Zh. Eksp. Teor. Fiz. 64 (1973) 48].

[18] S. L. Detweiler, Phys. Rev. D 22 (1980) 2323.

[19] O. Klein, Z. Phys. 53 (1929) 157. 
[20] W. Unruh, Phys. Rev. Lett. 31 (1973) no.20, 1265.

[21] S. Chandrasekhar, Proc. Roy. Soc. Lond. A 349 (1976) 571.

[22] H. Kim, JCAP 0811 (2008) 007 [hep-th/9706010].

[23] R. Brito, V. Cardoso and P. Pani, Lect. Notes Phys. 906 (2015) pp.1 [arXiv:1501.06570 [gr-qc]].

[24] J. D. Bekenstein, Phys. Rev. D 7 (1973) 949.

[25] J. D. Bekenstein, Phys. Rev. D 7 (1973) 2333.

[26] R. Penrose and R. M. Floyd, Nature 229 (1971) 177.

[27] R. Vicente, V. Cardoso and J. C. Lopes, Phys. Rev. D 97 (2018) no.8, 084032, [arXiv:1803.08060 [gr-qc]].

[28] V. Cardoso and O. J. C. Dias, Phys. Rev. D 70 (2004) 084011 [hep-th/0405006].

[29] V. Cardoso, O. J. C. Dias, J. P. S. Lemos and S. Yoshida, Phys. Rev. D 70 (2004) 044039 Erratum: [Phys. Rev. D 70 (2004) 049903] [hep-th/0404096].

[30] O. J. C. Dias, Phys. Rev. D 73 (2006) 124035, [hep-th/0602064].

[31] V. Cardoso, O. J. C. Dias and S. Yoshida, Phys. Rev. D 74 (2006) 044008 [hep-th/0607162].

[32] J. G. Rosa, JHEP 1006 (2010) 015 [arXiv:0912.1780 [hep-th]].

[33] S. Hod, Phys. Lett. B 708 (2012) 320 [arXiv:1205.1872 [gr-qc]].

[34] R. Li, Phys. Lett. B 714 (2012) 337 [arXiv:1205.3929 [gr-qc]].

[35] J. C. Degollado and C. A. R. Herdeiro, Phys. Rev. D 89 (2014) no.6, 063005 [arXiv:1312.4579 [gr-qc]].

[36] C. A. R. Herdeiro, J. C. Degollado and H. F. Rúnarsson, Phys. Rev. D 88 (2013) 063003 [arXiv:1305.5513 [gr-qc]].

[37] S. Hod, Phys. Lett. B 736 (2014) 398 [arXiv:1412.6108 [gr-qc]].

[38] S. R. Green, S. Hollands, A. Ishibashi and R. M. Wald, Class. Quant. Grav. 33 (2016) no.12, 125022 [arXiv:1512.02644 [gr-qc]].

[39] S. R. Dolan, S. Ponglertsakul and E. Winstanley, Phys. Rev. D 92 (2015) no.12, 124047 [arXiv:1507.02156 [gr-qc]].

[40] S. Hod, Phys. Lett. B 761 (2016) 326 [arXiv:1612.02819 [gr-qc]].

[41] C. Herdeiro, V. Paturyan, E. Radu and D. H. Tchrakian, Phys. Lett. B 772 (2017) 63 [arXiv:1705.07979 [gr-qc]].

[42] S. Hod, Phys. Lett. B 786 (2018) 217 Erratum: [Phys. Lett. B 796 (2019) 256] [arXiv:1808.04077 [gr-qc]].

[43] S. R. Dolan, Phys. Rev. D 98 (2018) no.10, 104006 [arXiv:1806.01604 [gr-qc]].

[44] O. J. C. Dias and R. Masachs, Class. Quant. Grav. 35 (2018) no.18, 184001 [arXiv:1801.10176 [gr-qc]].

[45] K. Destounis, Phys. Rev. D 100 (2019) no.4, 044054 [arXiv:1908.06117 [gr-qc]].

[46] J. H. Huang, W. X. Chen, Z. Y. Huang and Z. F. Mai, Phys. Lett. B 798 (2019) 135026 [arXiv:1907.09118 [gr-qc]].

[47] R. Li, Y. Zhao, T. Zi and X. Chen, Phys. Rev. D 99 (2019) no.8, 084045.

[48] T. Johannsen, Class. Quant. Grav. 33 (2016) no.12, 124001 [arXiv:1602.07694 [astro-ph.HE]].

[49] M. Isi, M. Giesler, W. M. Farr, M. A. Scheel and S. A. Teukolsky, Phys. Rev. Lett. 123 (2019) no.11, 111102 [arXiv:1905.00869 [gr-qc]].

[50] M. Khodadi, A. Allahyari, S. Vagnozzi and D. F. Mota, JCAP 2009 (2020) 026 [arXiv:2005.05992 [gr-qc]].

[51] C. A. R. Herdeiro, E. Radu and H. Runarsson, Phys. Rev. D 92 (2015) no.8, 084059 [arXiv:1509.02923 [gr-qc]].

[52] C. Herdeiro, E. Radu and H. Rúnarsson, Class. Quant. Grav. 33 (2016) no.15, 154001 [arXiv:1603.02687 [gr-qc]].

[53] C. A. R. Herdeiro and E. Radu, Phys. Rev. Lett. 119 (2017) no.26, 261101 [arXiv:1706.06597 [gr-qc]].

[54] C. A. R. Herdeiro and E. Radu, Eur. Phys. J. C 80 (2020) no.5, 390 [arXiv:2004.00336 [gr-qc]].

[55] A. Rahmani, M. Khodadi, M. Honardoost and H. R. Sepangi, Nucl. Phys. B 960 (2020) 115185 [arXiv:2009.09186 [gr-qc]].

[56] S. Capozziello and V. Faraoni, Beyond Einstein Gravity: A Survey of Gravitational Theories for Cosmology and Astrophysics, Springer, (2011).

[57] P. Pani, C. F. B. Macedo, L. C. B. Crispino and V. Cardoso, Phys. Rev. D 84 (2011) 087501 [arXiv:1109.3996 
[gr-qc]].

[58] B. Kleihaus, J. Kunz and E. Radu, Phys. Rev. Lett. 106 (2011) 151104 [arXiv:1101.2868 [gr-qc]].

[59] T. Delsate, C. Herdeiro and E. Radu, Phys. Lett. B 787 (2018) 8 [arXiv:1806.06700 [gr-qc]].

[60] P. V. P. Cunha, C. A. R. Herdeiro and E. Radu, Phys. Rev. Lett. 123 (2019) no.1, 011101 [arXiv:1904.09997 [gr-qc]].

[61] V. Cardoso, I. P. Carucci, P. Pani and T. P. Sotiriou, Phys. Rev. D 88 (2013) 044056 [arXiv:1305.6936 [gr-qc]].

[62] V. Cardoso, I. P. Carucci, P. Pani and T. P. Sotiriou, Phys. Rev. Lett. 111 (2013) 111101 [arXiv:1308.6587 [gr-qc]].

[63] A. N. Aliev, JCAP 1411 (2014) 029 [arXiv:1408.4269 [hep-th]].

[64] C. Y. Zhang, S. J. Zhang and B. Wang, JHEP 1408 (2014) 011 [arXiv:1405.3811 [hep-th]].

[65] O. Fierro, N. Grandi and J. Oliva, Class. Quant. Grav. 35 (2018) no.10, 105007 [arXiv:1708.06037 [hep-th]].

[66] M. F. Wondrak, P. Nicolini and J. W. Moffat, JCAP 1812 (2018) 021 [arXiv:1809.07509 [gr-qc]].

[67] T. Kolyvaris, M. Koukouvaou, A. Machattou and E. Papantonopoulos, Phys. Rev. D 98 (2018) no.2, 024045 [arXiv:1806.11110 [gr-qc]].

[68] A. Rahmani, M. Honardoost and H. R. Sepangi, Phys. Rev. D 101 (2020) no.8, 084036 [arXiv:2002.01663 [gr-qc]].

[69] M. Khodadi, A. Talebian and H. Firouzjahi, arXiv:2002.10496 [gr-qc].

[70] C. Y. Zhang, S. J. Zhang, P. C. Li and M. Guo, JHEP 2008 (2020) 105 [arXiv:2004.03141 [gr-qc]].

[71] P. Liu, C. Niu and C. Y. Zhang, arXiv:2005.01507 [gr-qc].

[72] V. A. Kostelecky, Phys. Rev. D 69 (2004) 105009 [hep-th/0312310].

[73] V. A. Kostelecky and R. Potting, Phys. Lett. B 381 (1996) 89 [hep-th/9605088].

[74] D. Colladay and V. A. Kostelecky, Phys. Rev. D 55 (1997) 6760 [hep-ph/9703464].

[75] D. Colladay and V. A. Kostelecky, Phys. Rev. D 58 (1998) 116002 [hep-ph/9809521].

[76] S. R. Coleman and S. L. Glashow, Phys. Rev. D 59 (1999) 116008 [hep-ph/9812418].

[77] M. D. Seifert, Phys. Rev. D 81 (2010) 065010 [arXiv:0909.3118 [hep-ph]].

[78] J. Páramos and G. Guiomar, Phys. Rev. D 90 (2014) no.8, 082002 [arXiv:1409.2022 [astro-ph.SR]].

[79] D. Capelo and J. Páramos, Phys. Rev. D 91 (2015) no.10, 104007 [arXiv:1501.07685 [gr-qc]].

[80] R. Casana, A. Cavalcante, F. P. Poulis and E. B. Santos, Phys. Rev. D 97 (2018) no.10, 104001 [arXiv:1711.02273 [gr-qc]].

[81] R. Oliveira, D. M. Dantas, V. Santos and C. A. S. Almeida, Class. Quant. Grav. 36 (2019) no.10, 105013 [arXiv:1812.01798 [gr-qc]].

[82] A. Ovgün, K. Jusufi and I. Sakalli, Annals Phys. 399 (2018) 193 [arXiv:1805.09431 [gr-qc]].

[83] A. Övgün, K. Jusufi and I. Sakalli, Phys. Rev. D 99 (2019) no.2, 024042 [arXiv:1804.09911 [gr-qc]].

[84] S. Kanzi and İ. Sakall, Nucl. Phys. B 946 (2019) 114703 [arXiv:1905.00477 [hep-th]].

[85] C. Ding, C. Liu, R. Casana and A. Cavalcante, Eur. Phys. J. C 80 (2020) no.3, 178 [arXiv:1910.02674 [gr-qc]].

[86] C. Liu, C. Ding and J. Jing, arXiv:1910.13259 [gr-qc].

[87] W. D. R. Jesus and A. F. Santos, Mod. Phys. Lett. A 34 (2019) no.22, 1950171 [arXiv:1903.09316 [gr-qc]].

[88] Z. Li and A. Övgün, Phys. Rev. D 101 (2020) no.2, 024040 [arXiv:2001.02074 [gr-qc]].

[89] W. D. R. Jesus and A. F. Santos, Int. J. Mod. Phys. A 35 (2020) no.09, 2050050 [arXiv:2003.13364 [gr-qc]].

[90] S. Chen, M. Wang and J. Jing, JHEP 2007 (2020) 054 [arXiv:2004.08857 [gr-qc]].

[91] S. Hod, Phys. Lett. B 713 (2012) 505 [arXiv:1304.6474 [gr-qc]].

[92] G. Creci, S. Vandoren and H. Witek, Phys. Rev. D 101 (2020) no.12, 124051 [arXiv:2004.05178 [gr-qc]]. 